\title{
Uncertainty assessment of coupled Digital Image Correlation and Particle Image Velocimetry for fluid-structure interaction wind tunnel experiments
}

\author{
L. Marimon Giovannetti ${ }^{\mathrm{a}}$, J. Banks ${ }^{\mathrm{a}}$, S. R. Turnock ${ }^{\mathrm{a}}$, S. W. Boyd $\mathrm{d}^{\mathrm{a}}$ \\ ${ }^{a}$ Faculty of Engineering and the Environment, Fluid Structure Interaction Group, \\ University of Southampton, UK
}

\begin{abstract}
The development of advanced composite structures for maritime and aerospace applications requires the ability to quantify their actual performance under known fluid loads. One example is the need to investigate the differences in fluid-structure response of passive adaptive composite structures. A wind tunnel based method is used to quantify the structural behaviour, and fluid response, of a flexible aerofoil under fluid loading. The technique measures the deflection of the structure, with high speed stereoscopic Digital Image Correlation (DIC). The tip vortex position is measured using high resolution stereoscopic Particle Image Velocimetry (PIV). The accuracy of the two full-field optical measurement systems is quantified and the effect of optical interactions is assessed. A flexible NACA0015 rectangular plan-form aerofoil of $0.9 \mathrm{~m}$ span and aspect ratio of two is subjected to aerodynamic loading within a closed circuit wind tunnel. The wind speed was varied from 10 to $25 \mathrm{~m} / \mathrm{s}$ within a $3.5 \mathrm{~m} \times 2.4 \mathrm{~m}$ working section. The structural response is measured simultaneously with the fluid flow field around the tip vortex. The tip vortex core, which moved by $\approx 62 \mathrm{~mm}$ at the highest wind speed, is directly compared to the deformation of the structure, which deflected by $\approx$ $58 \mathrm{~mm}$. A maximum foil twist of $\approx 0.6 \mathrm{deg}$ was observed. The DIC accuracy is evaluated in static and transient conditions for translational and rotational movement. The DIC maximum error for translations, greater than or equal to $0.5 \mathrm{~mm}$, is less than $3 \%$ and less than $0.6 \%$ in dynamic motions. The DIC total error for rotations is less than $5 \%$ in static motions and $1 \%$ in
\end{abstract}

Email address: L.Marimon-Giovannetti@soton.ac.uk (L. Marimon Giovannetti) 
dynamic rotations. The PIV uncertainty is quantified a posteriori providing the errors due to the correlation algorithm and the experimental setup. The mean in-plane velocity component uncertainties in the vortex region varied between $1.2 \%$ and $3.5 \%$ depending on flow speed $(\approx 0.1 \mathrm{px})$ around the vortex structure. The mean out-of-plane velocity uncertainty around the vortex varies between $2 \%$ and $3.3 \%$ depending on flow speed.

Keywords: Fluid Structure Interaction, Uncertainty analysis, Digital Image Correlation, Particle Image Velocimetry

\section{Introduction}

Analytical solutions do not exist for most FSI problems (de Borst et al., 2013; Hou et al., 2012). Likewise, it has proved challenging to acquire experimental measures of dynamic coupling between applied fluid loading and a structure response. As a result, research in this area has mainly focused on coupled Computational Fluid Dynamics (CFD) and structural Finite Element Analysis (FEA) simulations. Even though numerical studies have been extensive, especially in recent years with the increase in computer power, there is a lack of experimental validation cases for FSI problems and, for the limited cases that there are, the uncertainty in measurements is often unknown.

The advent of composite structures provides the designer with the potential to tune the response of the structure to the load applied. Fedorov (2012) presents a numerical and experimental approach where the effects of loads on a composite bend-twist full-scale wind turbine blade are measured. In particular they measured deflection and twist using Digital Image Correlation (DIC), however the uncertainty is not stated. It also lacks the dynamic coupling from the aerodynamic forces, as a known hydraulic load was applied whereas the aerodynamic force will actually change due to fluid induced deformation and twist.

High speed cameras and laser Doppler vibrometer were employed to measure the pitch motion of a flexible hydrofoil and the areas of cavitation (Ducoin et al., 2012). These experiments present the displacement and pitch angle for the tip section of a two-dimensional hydrofoil under real flow conditions. This study provides useful validation material for cavitation simulations including structural deformation but does not provide the hydrodynamic forces or flow field information to assess non-cavitating CFD models. 
Malijaarsl and Kaminski (2015) present a review on the published studies on flexible propellers. The possibility of using composite propellers to reduce cavitation problems is addressed, but they identify a need for experiments to validate the hydro-elastic numerical simulations as well as measurements of the deformed shape of flexible propellers.

To assess the validity of numerical FSI simulations we require the ability to measure the influence of fluid load on the structural response. It is important this is conducted in a controlled manner to provide data with known uncertainties for comparison with numerical FSI simulations. CFD is often validated in isolation using flow field data captured with Particle Image Velocimetry (PIV) and measured aero-hydrodynamic forces (Jones et al., 2008). Similarly FEA models can be validated against full field deformation measurements acquired using DIC (Siddiqui, 2014). This work aims to quantify the uncertainty associated with a wind tunnel based experimental method that couples both DIC and PIV.

It is the ability to synchronise the two measurement techniques and to minimise any optical interference effects that are essential elements of the developed methodology. The coupling of the two measurement techniques is discussed and an experimental set-up that encompasses PIV and DIC is described and their individual errors are assessed. This set-up has been developed to minimise the measurement error while synchronising the two techniques. A flexible finite span composite aerofoil is used to demonstrate the FSI methodology that is expected to be applicable to aero and hydro elastic problems. This includes the synchronised measurement of large structural deformations, changes in angle of attack and associated tip vortex motions.

\section{Nomenclature}

$\begin{array}{ll}d_{p} & \text { seeding particle diameter } \\ f & \text { lens } f \text {-number } \\ N_{P} & \text { seeding particle density } \\ R_{e} & \text { Reynolds number } \\ \mathrm{U} & \text { PIV velocity in tunnel x-direction } \\ \mathrm{V} & \text { PIV velocity in tunnel y-direction } \\ V_{S} & \text { wind speed } \\ \mathrm{W} & \text { PIV velocity in tunnel z-direction } \\ \Delta t & \text { PIV delay }\end{array}$




$\begin{array}{ll}\alpha & \text { foil angle of attack } \\ \psi & \text { camera stereo angle } \\ u, v, w & \text { flow components associated with wind tunnel co- } \\ & \text { ordinate system } \\ x, y, z & \text { wind tunnel coordinate system }\end{array}$

\section{Background on full-field measuring techniques}

Digital Image Correlation (DIC) and Particle Image Velocimentry (PIV) are both full field, non-contact, light based optical techniques and have been extensively used in a wide variety of experiments separately since the 1980s (Raffel et al., 2007; Chen et al., 2013).

DIC is generally used to measure the strain or displacement of a structure subjected to load. It is a white light full-field non-contact measurement technique (Tang et al., 2012). It involves the use of Charged-Coupled Device (CCD) digital cameras that register a series of images of a surface on which a randomised speckle pattern is applied. From these images, the displacement of the specimen can be calculated using a correlation algorithm to determine the motion of the speckle pattern within the field of view (Rastogi and Erwin, 2012). This technique has been used at different scales, from high magnification (Crammond et al., 2013) to large scale structures (Mccormick and Lord, 2012). Within the DIC software, the speckle pattern is mapped to calculate it's deformation, allowing the displacements and strains of the underlying structure to be measured (Rastogi and Erwin, 2012).

DIC analysis of a foil structure within the working session of a wind tunnel was developed at the University of Southampton (Banks et al., 2015). This initial study showed that small deflections could be accurately measured but that the structure was too stiff to result in significant twist deformations.

In order to correctly measure the structural response of the flexible aerofoil, high-speed three-dimensional DIC (3D DIC) is used in a stereo configuration. The stereo angle between the two cameras controls the measurement accuracy of the out of plane deformation (Tang et al., 2012; Schreier et al., 2009). This was very important in the present research as the major mode of deformation was out-of-plane and the stereo angle was carefully considered with respect to the dimensional constraints of the wind tunnel (Reu, 2012; Wang et al., 2011). As demonstrated by Crammond et al. (2013), the variations in speckle pattern density and sizes influence the measurement ac- 
curacy. Therefore, it is possible to design a speckle pattern tailored for each experimental measure. Moreover, to guarantee accurate measures, the illumination intensity and light should remain stable during the experiments. A number of studies were performed on the accuracy of DIC measures of specimens subject to high temperatures and extreme lighting conditions. In these cases it is necessary to combine bandpass filters with monochromatic light (Grant et al., 2009; Pan et al., 2012; Guo et al., 2014). Those findings were brought together in the current research to be able to accurately measure the deflections of a specimen with a fixed speckle size and density under varying lighting conditions. The DIC measurement error can be assessed through the use of motorised stages that provide known displacements of a specimen (Banks et al., 2015).

PIV can be used to assess the fluid flow field at a given plane, illuminated by a sheet of laser light. It is a full-field, non-contact measurement technique that involves the use of CCD digital cameras to capture the reflected light of small seeding particles included in the flow. A camera records two images in rapid succession (with a given separation time, $\Delta t$ ) so that the light pattern from the seeding particles is recorded in both images. The images are divided into small areas allowing a cross-correlation algorithm to determine the displacement vector of each interrogation area from one image to the next (Raffel et al., 2007). A single camera can be used to determine the velocity components within the plane of the laser sheet. However, significant errors can be observed if the optical axis of the camera is not perpendicular to the laser sheet. Unavoidable errors can also be introduced by out-of-plane particle motions observed as in-plane motions due to the perspective transformation. By using two cameras in a stereoscopic system the out-of-plane motion can be used to determine all three velocity components on the laser sheet plane. This minimises errors associated with misalignment and perspective transformation. In stereo PIV the two cameras are positioned so that their optical axes intersect at the midpoint of the field of view (Prasad and Jensen, 1995). However, in order to allow a good focus over the entire field of view the use of the Scheimpflug condition is required (Prasad, 2000). This adjusts the angle of the camera sensor relative to the lens in order to align the depth of field to the laser sheet.

The accuracy of the velocity vectors from PIV measurements can depend on many different factors. Systematic (or bias) errors can be caused by limitations of the correlation algorithm, calibration errors and particle images of one pixel or less causing peak locking. Residual (or random) errors can be 
typically caused by camera noise, high background image intensity reducing the contrast of particle images, defocused particle images, seeding density, particle image shift and out of plane motion reducing particle image pairs from one frame to the next (Sciacchitano et al., 2015). Historically these uncertainties could only be assessed if you already know the velocity field being measured or by using synthetic data. However, recently different approaches have been proposed for a posteriori uncertainty quantification. The correlation statistics method was proposed by Wieneke (2015) and is implemented within the commercial PIV software DaVis (LaVision, 2016). This approach uses the converged solution of the displacement field to de-warp the second image back onto the first image. If an exact displacement field was calculated each particle image would be mapped directly onto its position in the first image creating a symmetric correlation peak. Any differences between the de-warped image and the first image are due to errors in the displacement field and create a non-symmetric correlation peak. A statistical analysis of how each pixel contributes to the correlation peak shape allows the vector uncertainty to be determined. This method was shown to accurately determine various sources of error in synthetic data including: out-of plane motion, seeding density, particle image size, velocity gradients across interrogation windows and image noise. A comparison of different a posteriori uncertainty quantification techniques concluded that the correlation statistics approach was the most accurate method assessed, typically reproducing real experimental error with $85 \%$ accuracy (Sciacchitano et al., 2015).

PIV has been used in literature for simultaneous measures of temperature and different flow components using different light sources and the corresponding bandpass filters (Post et al., 1994; Funatani et al., 2004). Those applications are particularly interesting in the simultaneous measures of DIC and PIV proposed in the current investigations due to the need to isolate different optical systems.

In order to make comparisons between different flow fields it is often useful to characterise the fluid structures using post-processing of the calculated velocity vector field. One such example is being able to locate the centre of a vortex structure. The basic VORTFIND algorithm (Pemberton et al., 2002) can be applied to a transverse vector field on a plane perpendicular to the vortex direction. Each vector is assigned to a velocity direction sector of the flow field based on the angle between the vector and a reference axis. A vortex centre will be surrounded by vectors from each direction sector as the flow rotates about that point. Therefore, if you rank all the vectors by 
their proximity to different sectors you can determine how close they are to a vortex centre. This approach allows the position of a single vortex structure to be located and is simple to implement, but can be extended to cope with multiple vortex structures if required (Phillips and Turnock, 2013).

\section{Experimental set-up}

The wind tunnel provides a controlled environment where it is possible to accurately describe a FSI problem. In order to measure the aerodynamic loading, the structural response and the fluid behaviour, the two optical measurement techniques, DIC and PIV, are required to be synchronised with a force acquisition system.

The experiments were conducted in the $3.5 \mathrm{~m}$ x $2.4 \mathrm{~m} \mathrm{R}$. J. Mitchell closed circuit wind tunnel at the University of Southampton. Its turbulence intensity levels are less than $0.2 \%$ (Castro, 2001).

The investigated aerofoil is a NACA0015 section containing a load-carrying beam, an aerofoil-shaped foam-rib structure and a layer of Mylar to transfer the aerodynamic loading to the foam and the beam, see Figure 1. The load-carrying beam is made of a sandwich structure (where the two skins are unidirectional carbon fibre and the core is Aluminium 6082). This structure was chosen for its simplicity, reproducibility and flexibility to provide a test case that can be easily replicated in a numerical environment.

The manufacturing process of the sandwich structure involved the use of unidirectional (UD) prepreg carbon fibre SE 84LV (Gurit, 2015b) and a toughened epoxy adhesive film SA 80 (Gurit, 2015a) to fully bond the carbon skins to the 6082 aluminium. The manufacturing process was carried out laying-up the UD carbon fibres at the desired ply angle (zero degrees) and then curing the sandwich structure under vacuum at 2 bar and $120^{\circ} \mathrm{C}$ for one hour. This structure was chosen so that different fibre lay-ups could be tested in the wind tunnel, whilst maintaining the same aerodynamic shape.

The manufacturing process for the aerofoil structure used the hot-wire technique to cut foam to the desired shape. The foam sections (including the leading edge, trailing edge and ribs) are joined together by means of a two-component epoxy adhesive and the Mylar is added to form an outer skin. The aerofoil principal dimensions can be seen in Figure 1, its thickness is $67.5 \mathrm{~mm}$ and its area $40500 \mathrm{~mm}^{2}$. The structure is designed to be able to deflect more than $10 \%$ of its span length and to withstand the wind tunnel aerodynamic loadings for a Reynolds Number $\left(R_{e}\right)$ of 750,000 , corresponding 
to the highest wind speed tested in the wind tunnel, as described in (Note, 2014).

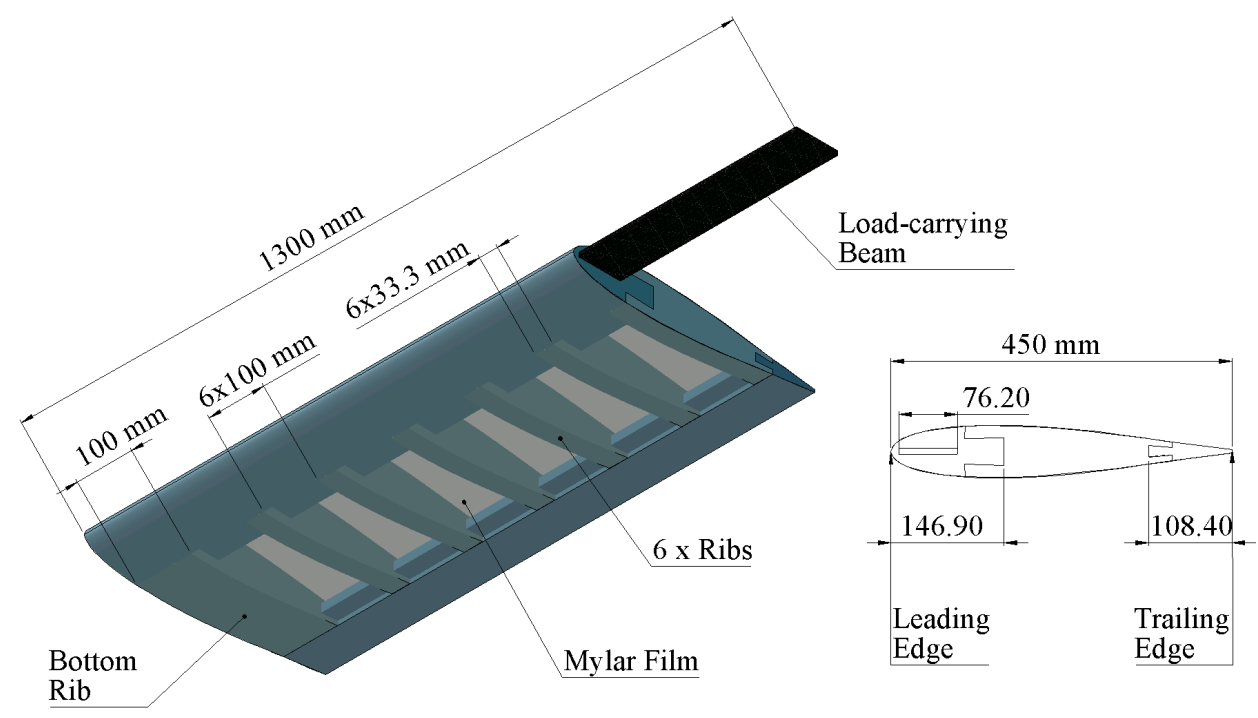

Figure 1: Tested specimen showing the load carrying carbon-aluminium beam, the foam rib structure and the Mylar sheet as well as the principal dimensions.

The aerodynamic forces on the NACA0015 foil are measured via a six component Nuntem load cell balance, mounted on a turntable in the roof of the wind tunnel. The aerodynamic data was acquired at a sampling frequency of $1 \mathrm{kHz}$. At least two repeats were measured in order to assess the repeatability and the robustness of the aerodynamic results.

High-speed three-dimensional DIC is used to capture the structural response of the foil. Details of the set-up and equipment used are provided in Table 1 . The acquisition frame rate varied between 0.1 to $1 \mathrm{kHz}$. Two high speed SA3 Photron cameras were placed in the viewing room of the wind tunnel to avoid aerodynamic loading and vibrations on the cameras. All the mean DIC results are averaged over a large number of pictures, varying from 100 to 3000 images. In addition, 30 second recordings at $0.1 \mathrm{kHz}$ were captured to be able to describe the structural and fluid behaviour in the coupled DIC-PIV measurements.

Four high-powered LED lights (NILA Zaila) were mounted in the wind tunnel ceiling and floor to provide the required level of light to the DIC 
cameras. The greyscale histogram of the applied speckle pattern is assessed to achieve the correct level of diffused light (Crammond et al., 2013).

Table 1: DIC equipment and the setting used in the experiment.

\begin{tabular}{ll}
\hline Equipment & Set-up \\
\hline \hline & 2 high speed SA3 Photron \\
& Sensor size: $17.4 \times 17.4 \mathrm{~mm}$ \\
Camera & Pixel size: $17 \mu \mathrm{m}$ \\
& Resolution $(\max ): 1024 \times 1024$ pixels \\
& Exposure time: $2000 \mu \mathrm{s}$ \\
& Frame rate: $0.1-1 \mathrm{kHz}$ \\
\hline \multirow{2}{*}{ Lens } & Tokina $100 \mathrm{~mm} \mathrm{f} .8$ \\
& Aperture: $f-16$ \\
\hline \multirow{2}{*}{ Speckle pattern } & Speckle size: approx. 7 pixels \\
& Dimensions: $450 \times 450 \mathrm{~mm}$ \\
\hline
\end{tabular}

A stereoscopic PIV system was used to assess the on all three velocity components, on a vertical plane one chord downstream of the foil tip. Seeding particles were generated using two smoke machines, with the particle size and density varying with the period of smoke generation, the free stream velocity in the tunnel and the time allowed for even particle distribution. Table 2 describes the set-up and the equipment used in the PIV system. Two high-resolution LaVision Imager LX 29M PIV cameras were mounted on the wind tunnel ceiling 4 chords downstream of the trailing edge of the specimen for the PIV measurements. The acquisition frame rate for the PIV images was set to $2 \mathrm{~Hz}$ and the time between the two cross-correlation frames was adjusted for each wind speed to provide a constant particle displacement of approximately $1 / 4$ of the laser sheet thickness. The raw images were acquired and processed using the LaVision software DaVis 8.2. No image preprocessing was performed before the velocity vectors were calculated using a multi-pass stereo cross-correlation process decreasing in window size from $64 \mathrm{x} 64$ px to $48 \mathrm{x} 48 \mathrm{px}$, with a $75 \%$ overlap.

The Litron Bernoulli Laser was mounted below the wind tunnel working section, 1 chord downstream of the foils trailing edge. A 90 degree mirror and laser sheet optics were used to focus the sheet $1.3 \mathrm{~m}$ above the tunnel floor, i.e. corresponding to the location of the tip of the foil. The sheet thickness 
was estimated to be $2-4 \mathrm{~mm}$ thick but could not be reduced further using the available optics.

Figure 2 shows a schematic set-up of the two full-field measurement systems in the wind tunnel. The reference point about which the forces and moments are measured has been added and provides the coordinate system for the PIV data, presented in the tunnel axis system. 


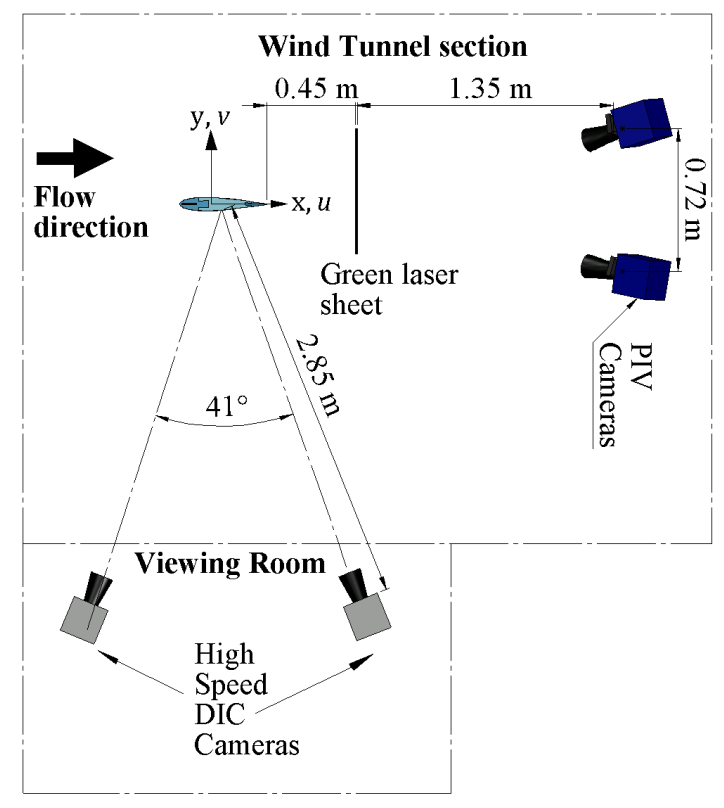

(a) Plan view

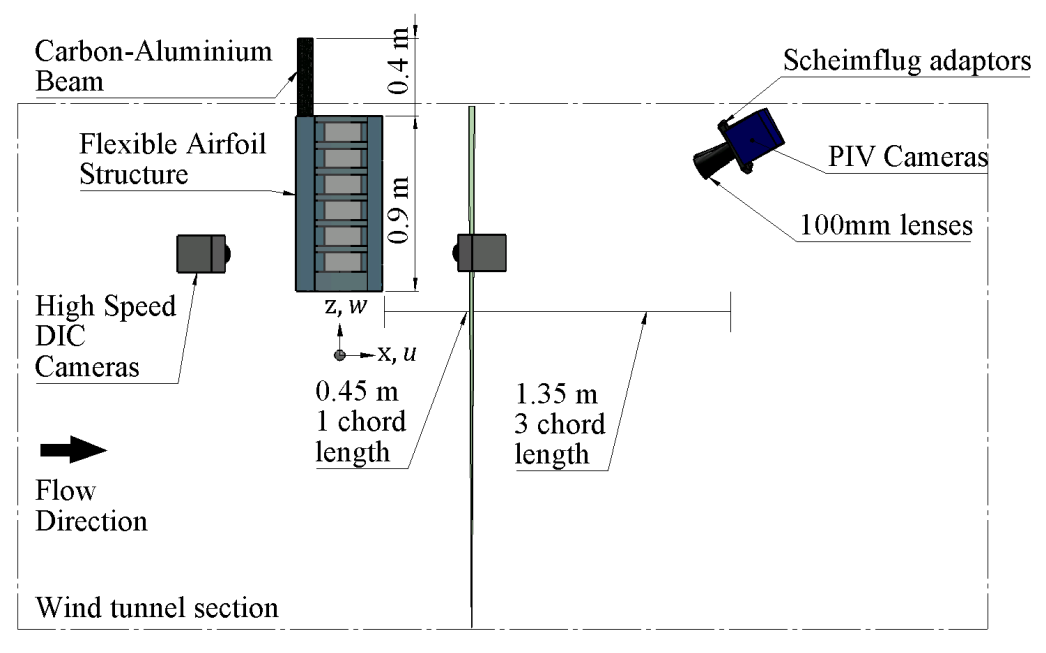

(b) Side view

Figure 2: DIC and PIV setup in the wind tunnel showing the principal dimensions and the used components. 
The reference point is located on the tunnel centreline $1.26 \mathrm{~m}$ below the tunnel ceiling, $0.31 \mathrm{~m}$ below the tip of the unloaded foil and $0.09 \mathrm{~m}$ downstream of the unloaded leading edge. The stereo angle accuracy is $\pm 0.5^{\circ}$ and the linear measures accuracy is $\pm 2.5 \mathrm{~mm}$.

In order to correctly focus the stereo PIV cameras, two Scheimflug adaptors were mounted on the PIV cameras. These adaptors correct the limited depth of field over the field of view, given by the stereo and the pitch angles of the cameras, tilting the image plane to align with the plane of the laser sheet, in accordance with the Scheimflug criterion (Prasad, 2000).

Table 2: PIV performance table showing the equipment and the setting used.

\begin{tabular}{|c|c|}
\hline Equipment & Set-up \\
\hline Camera & $\begin{array}{l}2 \text { ImagerLX } 29 \mathrm{M} \text { (LaVision) } \\
\text { Sensor size: } 36.3 \times 24.2 \mathrm{~mm} \\
\text { Pixel size: } 5.5 \mu \mathrm{m} \\
\text { Resolution }(\max ):(6.4 \times 4.4) \times 10^{3} \text { pixels } \\
\text { Exposure time: } 1 / 500 \mu \mathrm{s} \\
\text { Frame rate: } 2 \mathrm{~Hz} \\
\text { Stereo angle: } \approx 27 \mathrm{deg} . \\
\text { Pitch angle: } \approx 25 \mathrm{deg} . \\
\Delta t=100-50-40 \mu \mathrm{s} \text { for } V_{S}=10-20-25 \mathrm{~m} / \mathrm{s}\end{array}$ \\
\hline Lens & $\begin{array}{l}\text { Tokina } 100 \mathrm{~mm} \mathrm{f} 2.8 \\
\text { Aperture: } f \text {-4 } \\
\text { Depth of field: } 16 \mathrm{~mm}\end{array}$ \\
\hline Laser & $\begin{array}{l}\text { Litron Bernoulli Nd: YAG PIV Laser } \\
\text { Wavelength: } 532 \mathrm{~nm} \\
\text { Output energy: } 200 \mathrm{~mJ}\end{array}$ \\
\hline PIV processing & $\begin{array}{l}\text { Software: DaVis } 8.2 \\
\text { Area of interest: Y }(-260 \text { to } 140 \mathrm{~mm}) \mathrm{Z}(25.5 \text { to } 145.5 \mathrm{~mm}) \\
\text { Digital resolution: } 9.8 \mathrm{px} \mathrm{mm}-1 \\
\text { Initial interrogation area: } 64 \times 64 \mathrm{px} \\
\text { Final interrogation area: } 48 \times 48 \mathrm{px}(75 \% \text { overlap })\end{array}$ \\
\hline
\end{tabular}

Both the DIC and PIV systems were calibrated using a LaVision type31 calibration plate that covers the majority of the area of interest of the two systems. The HS DIC system was controlled by a LaVision High speed 
controller, while the PIV system was controlled by a LaVision programmable timing uint,PTU9 (LaVision, 2015). The recording frequency of the DIC system was set to be a multiple of the PIV system. The DIC system was set to an external trigger, which was provide by the PIV camera trigger. This ensured that the DIC and PIV images were synchronised. The trigger and image clock signals were recorded by the force and moment data acquisition system to relate the aerodynamic loading to each recorded full-field image.

It was necessary to ensure optical isolation between the two full field measurement techniques: the white light DIC and the laser illuminated PIV. Therefore, two low-pass $532 \mathrm{~nm} \pm 10 \mathrm{~nm}$ filters were applied to the PIV cameras to allow them to only capture the laser wavelength. In addition, the LED lights were covered with magenta gel filters to remove any green light illumination of the foil structure that might be detected by the PIV cameras. This resulted in the diffused light detected by the high-speed DIC cameras only containing two-thirds of the original white light histogram. The adopted method provided enough light that the aperture and exposure settings of the cameras did not need to be adjusted. This ensured that the comparison of the results between DIC in isolation and synchronised DIC/PIV includes the introduction only of errors deriving from the addition of the filters.

Figure 3 shows pictures from the wind tunnel working section during the calibration process and it shows both the DIC and PIV illumination used for coupled DIC-PIV runs. 


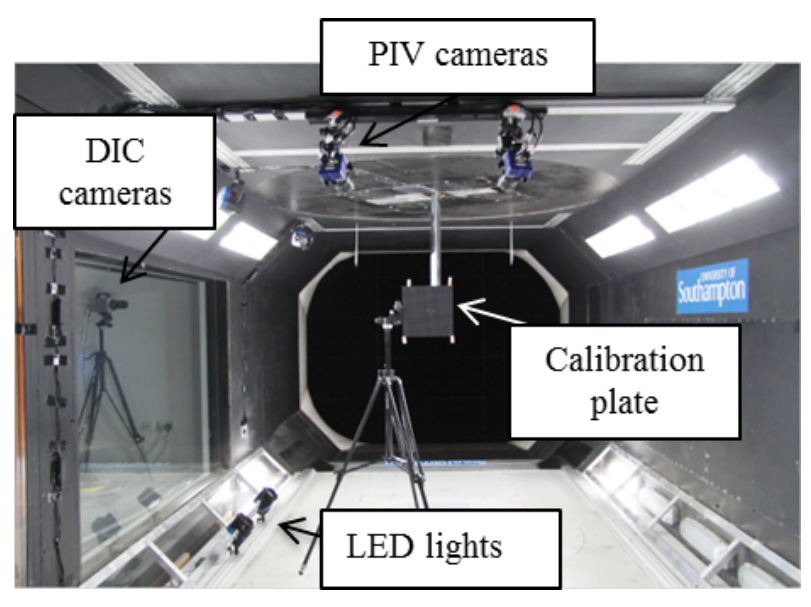

(a) Wind tunnel section during PIV calibration process

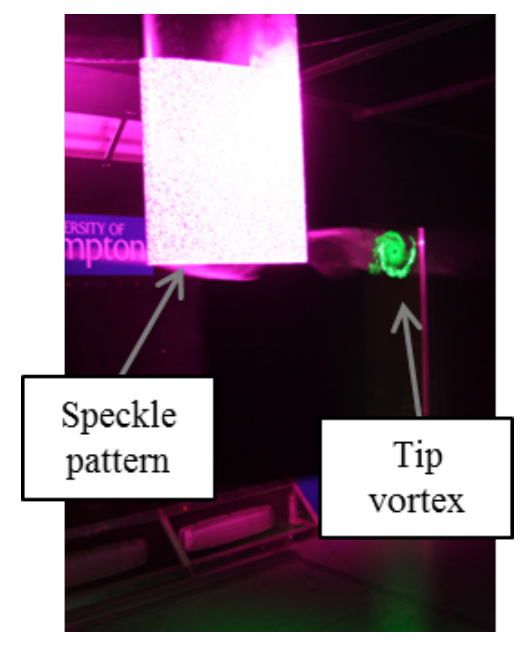

(b) DIC-PIV coupled illumination

Figure 3: Wind tunnel set-up.

\section{Measurement accuracy of high speed Digital Image Correlation}

In order to fully capture the structural response of the aerofoil, as concluded by Banks et al. (2015), it was necessary to acquire the DIC images with high-speed cameras. Given the fixed dimensions of the wind tunnel and the level of accuracy needed for relatively large out-of-plane deflections, the maximum achievable stereo angle $(\psi)$ of $41^{\circ}$ was used. The acquisition frequency varied between 0.1 to $1 \mathrm{kHz}$ depending on the length of recorded time.

The accuracy of the DIC was quantified against Standa high-precision motorised stages in both translation (8MT175-50 motorised linear stage) and rotation (8MR174-11 motorised rotation stage) in static and transient conditions. The motorised translation stage has an accuracy of $2.5 \mu \mathrm{m}$ and can travel at a speed of $5 \mathrm{~mm} / \mathrm{s}$. In static conditions the DIC out-of-plane accuracy has been tested for a range of translations from $0.1-50 \mathrm{~mm}$, as can be seen in Figure 4. The percentage error is calculated between the measured values of the DIC and the motions input to the motorised stage as:

$$
\%_{\text {error }}=\left|\frac{D I C_{\text {measure }}-\text { Stage }_{\text {translation }}}{\text { Stage }_{\text {translation }}}\right| \times 100
$$


The results are presented in pixel shift as well as mm offset to enable comparison with results obtained from cameras of different resolutions. The accuracy of the results for small out-of-plane deflections (i.e. 0.1-0.25 mm) presents large errors due to the chosen stereo angle. The stereo angle provides a tradeoff in accuracy for in-plane and out-of-plane translations (Reu, 2012; Wang et al., 2011). In order to decrease error in relatively small out-of-plane rigid body motions, the stereo angle should be increased. Figure 4(b) shows that for deformations equal to or greater than $0.5 \mathrm{~mm}$ the error is less than $3 \%$. 


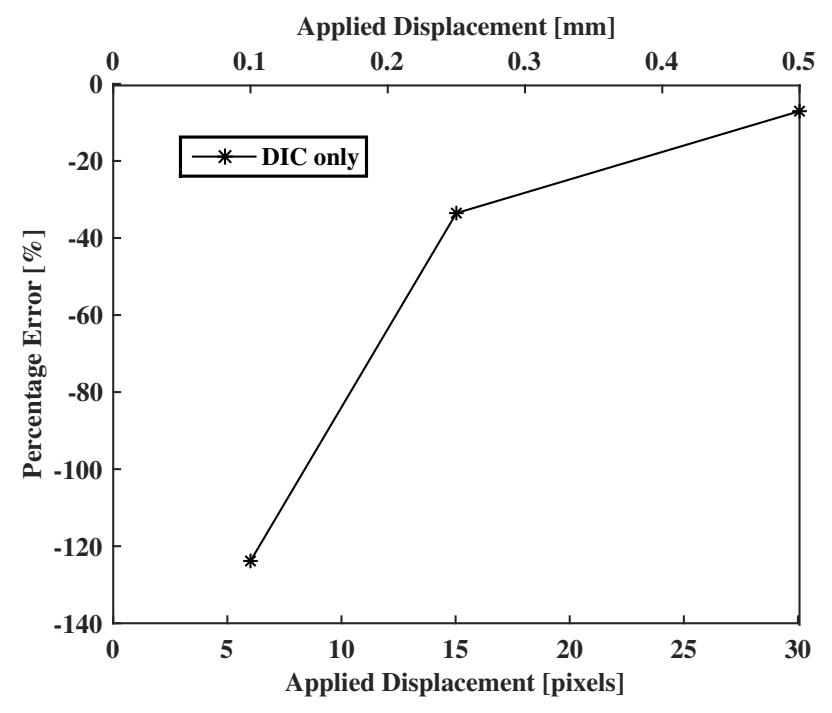

(a) 0.1 to $0.5 \mathrm{~mm}$ rigid body motion

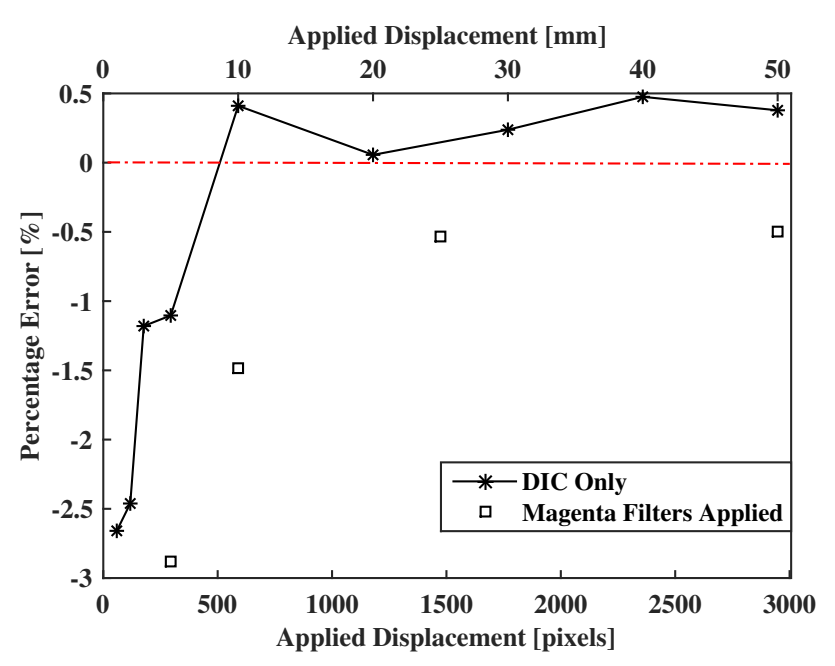

(b) $0.5-50 \mathrm{~mm}$ rigid body motion

Figure 4: Static out-of-plane displacement accuracy including the influence of the magenta filters applied on the LED lights. 
From Figure 4(b) it can be seen that the addition of the magenta gel filters on the LED lights, shifts the percentage error without changing its trend. This phenomenon is due to the change in the grey-scale histogram of the acquired images (Figure 5). As the exposure time and the lens aperture are not varied while the two measurement systems are captured, the histogram captured by the high-speed cameras for the same speckle pattern shows a shift toward the black end of the scale (i.e. 0 pixel intensity) and a lower pixel count. This produces the percentage error shift shown in Figure 4(b).

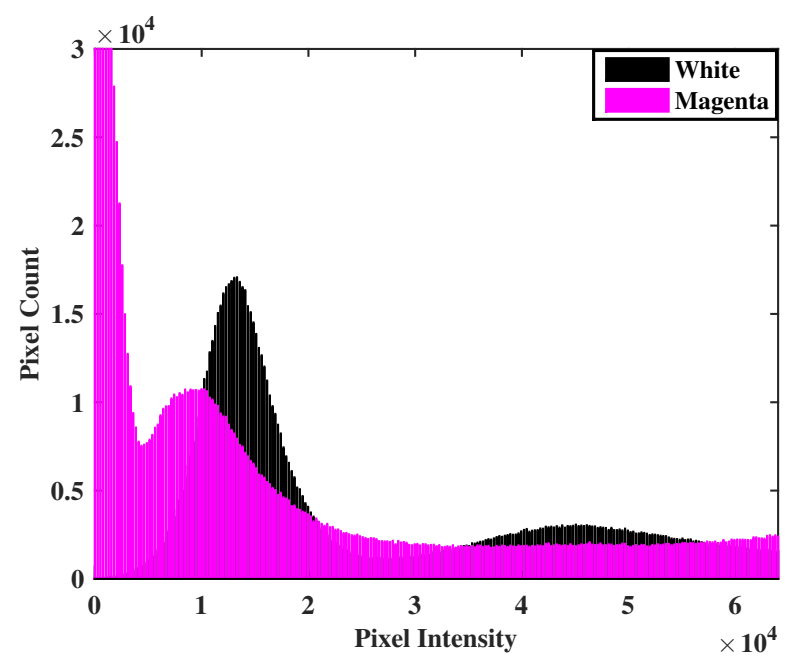

Figure 5: Histogram comparison between white light image and the addition of magenta filters.

In order to correctly assess the measurement accuracy in translation, the relative displacement error is also measured. This is achieved by comparing small relative displacements at the far end of the displacement range (i.e. calculating the relative error from $49 \mathrm{~mm}$ to 49.5 and $50 \mathrm{~mm}$ ). The percentage errors at the end of the translation range can be seen in Figure 6 compared to the small displacement captured at the near-end of the depth of field. For both cases, it can be seen that a rigid body motion of $1 \mathrm{~mm}$ yields a smaller percentage error. Moreover, it can be seen that the percentage error captured $49 \mathrm{~mm}$ away from the calibration plane is larger for both motions. This phenomenon might be due to the decrease in the relative stereo angle given by the larger out-of-plane distance of the specimen to the cameras. 
Nonetheless, the DIC accuracy has been shown to be acceptable for small displacements in the whole depth of field range.

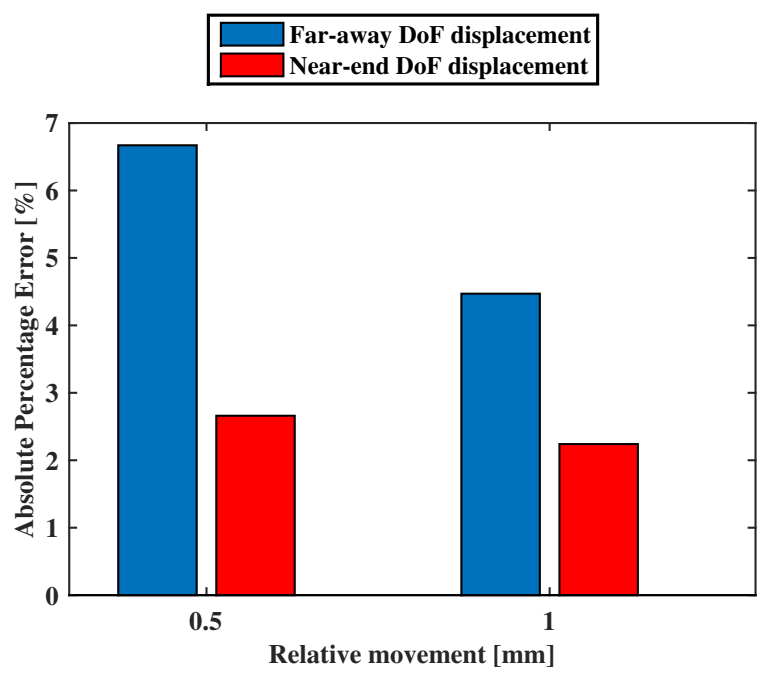

Figure 6: Percentage error comparison for Near- and Far-end depths of field rigid body motions of 0.5 and $1 \mathrm{~mm}$.

An assessment of the DIC accuracy for transient motions was conducted. The known rigid body motion was $5 \mathrm{~mm} / \mathrm{s}$ and the DIC measurement gave a rate of $4.97 \mathrm{~mm} / \mathrm{s}$, with a $\mathrm{R}^{2}$ value of $0 . \overline{9}$.

The accuracy of the DIC system is also assessed in rotation. The motorised rotation stage has an accuracy of \pm 0.1 degrees and can rotate at a speed of $15^{\circ} / \mathrm{s}$. The applied rotation varied from $0.1^{\circ}$ to $20^{\circ}$. This represents a realistic range of local angles of attack observed in the experiments and quantifies the accuracy of blade twist measurements obtained from the DIC measurements. Figure 7(a) presents the rotation measurement accuracy with and without the influence of the magenta gels on the DIC lights. As detected for the translation, the percentage error shifts with respect to the pure white light DIC, but the trend is consistent, showing that for larger applied angles the percentage error decreases. The addition of magenta filters changes the intensity profile of the speckle pattern as seen by the cameras. Comparing Figure 4(b) with Figure 7(a) it is possible to see that the error in rotation is decreased with respect to the white-light images measures. 


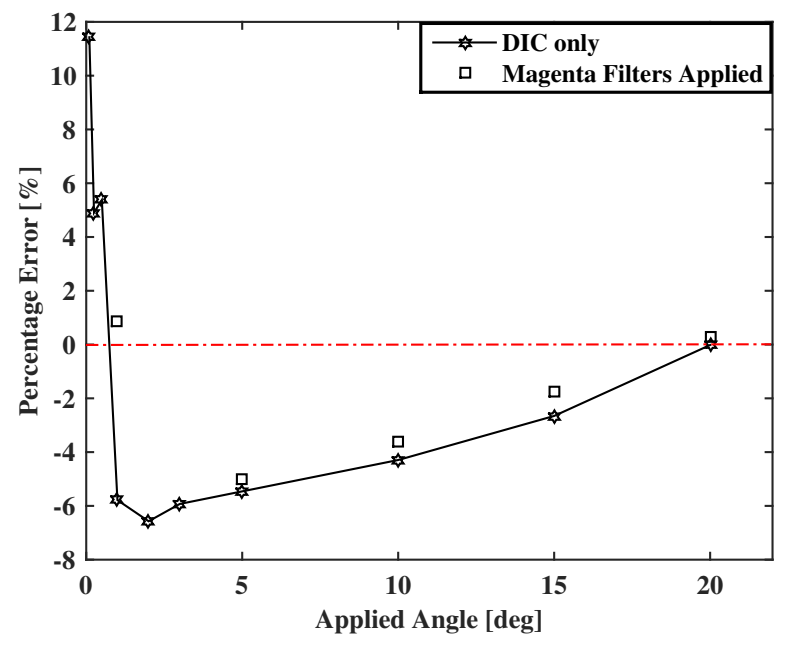

(a) Comparison of pure white light and the magenta gels statically

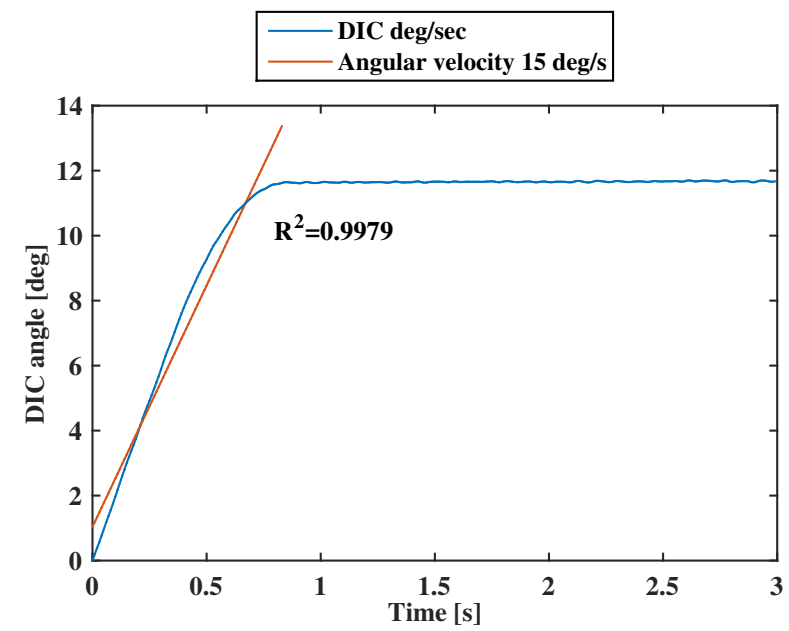

(b) DIC transient rotation accuracy over time

Figure 7: Accuracy of DIC measurements in rotation.

This phenomenon can be explained as the rotation measures take into 
consideration only two points at the sides of the specimen. In these locations the change in intensity level due to the filters is small. The translation data is averaged over the entire specimen and the change in intensity level is greater in the centre of the image. The percentage errors captured in rotation lie close to the accuracy of the rotation stage (0.1 degrees). The transient response of the rotation is also assessed. Figure $7(\mathrm{~b})$ shows the response captured by the high-speed DIC cameras for a change in angle of $15^{\circ} / \mathrm{s}$. The end of the rotation scale range is reached after $0.85 \mathrm{~s}$, where the DIC detected velocity becomes zero. Therefore, the angular velocity measured with the DIC system in the first $0.85 \mathrm{~s}$ of the recordings corresponds to $14.85^{\circ} / \mathrm{s}$.

A close inspection of the coupled DIC-PIV images showed that the seeding particles did not appear to be affecting the image quality of the DIC when the two measuring systems are synchronised.

\section{Measurement accuracy of high resolution Particle Image Velocimetry}

For each wind speed tested the raw images were inspected to determine some basic parameters that have been previously been linked to measurement error. The seeding particle diameter (dp) and density (Np) were estimated by eye within 4 interrogation windows near the centre of the area of interest, but not within the vortex core. This assessment was performed on an image acquired half way through the 120 image pairs and is presented in Table 3. This confirms that the smoke particles are not far from the optimum diameter of 2.5 px (Raffel et al., 2007). It is also observed that the recommended seeding density of 10 or more particles per interrogation window also appears to have been met (Raffel et al., 2007). The separation time between image pairs, $\Delta t$, is also provided, along with the associated mean and maximum inplane particle displacement in pixels and the axial particle displacement $(\mathrm{dx})$ in $\mathrm{mm}$. During the post processing of the results it was discovered that the separation time used for $10 \mathrm{~m} / \mathrm{s}$ was incorrectly set at $40 \mu \mathrm{s}$ instead of $100 \mu \mathrm{s}$. This significantly reduced the particle displacements observed, particularly near the edges of the area of interest, where the local in-plane displacements are smallest. 
Table 3: Seeding parameters for differnt free stream velocities.

\begin{tabular}{lccc}
\hline Free stream velocity $(\mathrm{m} / \mathrm{s})$ & 10 & 20 & 25 \\
\hline Particle size, $\mathrm{d}_{\mathrm{p}}(\mathrm{px})$ & $2-3$ & $2-4$ & $2-4$ \\
Particles per IW: $\mathrm{N}_{\mathrm{p}}$ & 11.5 & 13 & 10 \\
Seperation time $\Delta \mathrm{t}(\mu \mathrm{s})$ & 40 & 50 & 40 \\
Mean In-plane particle displ $(\mathrm{px})$ & 1.2 & 2.8 & 2.6 \\
Max In-plane particle displ $(\mathrm{px})$ & 2.7 & 6.5 & 6.7 \\
Particle X displ. Dx $(\mathrm{mm})$ & 0.4 & 1 & 1 \\
\hline
\end{tabular}

The uncertainty associated with the PIV measurements was assessed $a$ posteriori from correlation statistics within the software DaVis 8.2 (Wieneke, 2015 ), as described in section 2. The uncertainty of each vector is calculated for all of the 120 processed vector fields and averaged in time to provide the mean uncertainty over the area of interest for a given experimental condition. An example of the mean uncertainty bias and standard deviation for one of the in-plane velocity components is given in Figure 8. 


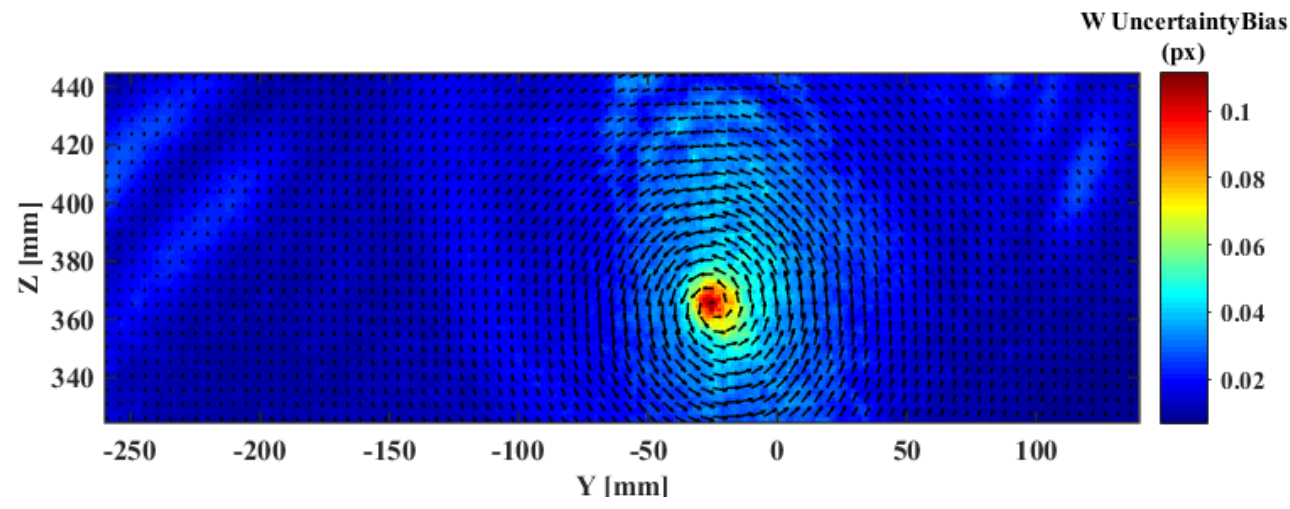

(a) Uncertainty bias

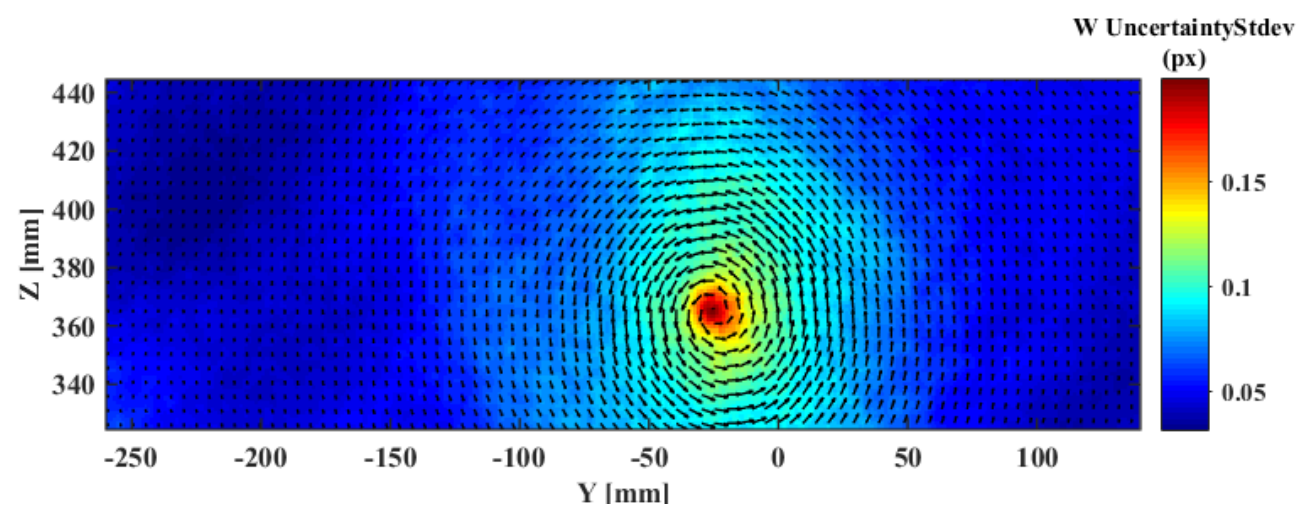

(b) Uncertainty Standard deviation

Figure 8: Vertical in-plane velocity component W, for 15 degrees angle of attack at 20 $\mathrm{m} / \mathrm{s}$. The in-plane velocity field is represented by vectors.

It can be observed that the systematic (bias) uncertainty is significantly smaller than the random (standard deviation) component. However both exhibit a similar distribution with maximum error located at the vortex core. Therefore, in future the total uncertainty of each velocity component has been presented as the root-mean-square of the bias and standard deviation. The measurement uncertainty over a range of free stream velocities can be observed in Figure 10. The in-plane velocity uncertainties are compared to the local in-plane velocity magnitude $|V+W|$ to provide the relative 
uncertainty for the transverse flow field. Likewise the uncertainty of the out-of-plane velocity $U$ is compared to the local $U$ velocity magnitude.

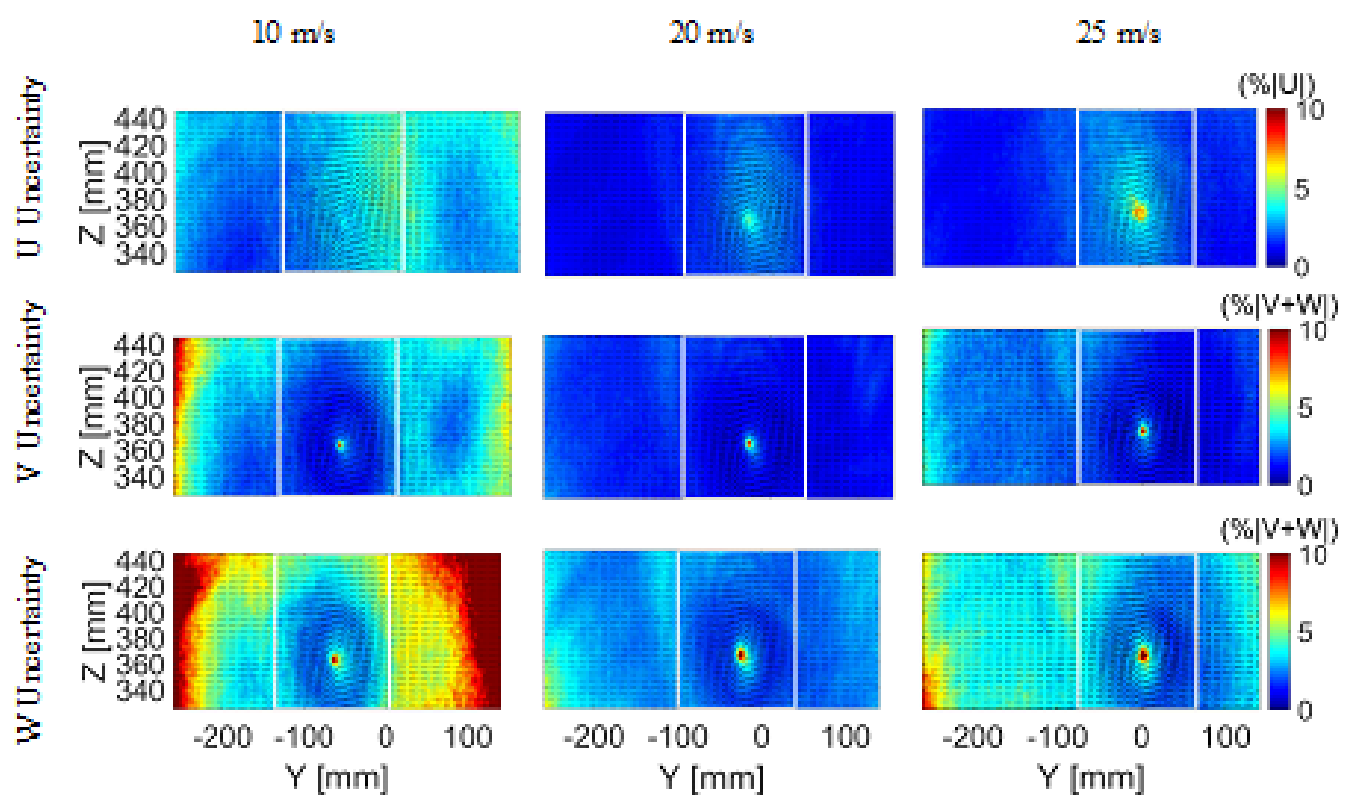

Figure 9: Velocity component uncertainty, as a percentage of either the local in-plane velocity magnitude or the local axial velocity, for different free stream velocity values. The area of interest around the vortex structure is defined by a white box.

It can be observed that the PIV uncertainty varies over the presented flow field. For instance the in-plane velocity uncertainties increase significantly as you approach the edge of the field of view. There are several potential causes for this. With stereoscopic PIV the focus tends to deteriorate away from the centre of the field of view due to imperfect correction of the depth of field. However as these trends are not observed in the out-of-plane uncertainty it is more likely to be the local in-plane velocity field which is causing this effect. The local in-plane velocity magnitude drops off rapidly as you move away from the vortex centre, reducing the pixel shift of particles in this region. As the in-plane velocity tends towards zero the relative uncertainty rapidly increases. It is important therefore that the uncertainty of PIV measurements are assessed within the region of the flow that you are most interested in. In an attempt to quantify the measurement uncertainty over a useful region a local area of interest has been identified around the vortex core, as depicted 
by white boxes in Figure 9. The averaged values for relative and absolute uncertainty within this area are presented in Table 4.

Table 4: Mean uncertainty values for each velotity component averaged over the area of interest depicted in Figure 9 .

\begin{tabular}{cccc}
\hline Free stream velocity $(\mathrm{m} / \mathrm{s})$ & 10 & 20 & 25 \\
\hline Mean U uncertainty & $3.30 \%|\mathrm{U}|$ & $2.04 \%|\mathrm{U}|$ & $2.51 \%|\mathrm{U}|$ \\
(Out-of-plane) & $0.13 \mathrm{px}$ & $0.21 \mathrm{px}$ & $0.25 \mathrm{px}$ \\
\hline Mean $\mathrm{V}$ uncertainty & $1.99 \%|\mathrm{~V}+\mathrm{W}|$ & $1.24 \%|\mathrm{~V}+\mathrm{W}|$ & $1.53 \%|\mathrm{~V}+\mathrm{W}|$ \\
(In-plane horizontal) & $0.03 \mathrm{px}$ & $0.05 \mathrm{px}$ & $0.06 \mathrm{px}$ \\
\hline Mean W uncertainty & $3.46 \%|\mathrm{~V}+\mathrm{W}|$ & $2.18 \%|\mathrm{~V}+\mathrm{W}|$ & $2.81 \%|\mathrm{~V}+\mathrm{W}|$ \\
(In-plane vertical) & $0.06 \mathrm{px}$ & $0.09 \mathrm{px}$ & $0.11 \mathrm{px}$ \\
\hline
\end{tabular}

In general the lowest measurement uncertainty was observed for the horizontal in-plane velocity component $\mathrm{V}$. Mean uncertainty values of less than $2 \%$ of the in-plane velocity magnitude were observed around the vortex region. This represents a mean absolute uncertainty of $0.06 \mathrm{px}$ or less. The mean relative uncertainty for the vertical in-plane velocity component $\mathrm{W}$ varies between $2.2 \%$ and $3.5 \%$ depending of the free stream velocity. This represents an absolute uncertainty of approximately $0.1 \mathrm{px}$ around the vortex core. In general the relative out of plane measurement uncertainty (for $U$ velocity) has larger absolute error values up to $0.25 \mathrm{px}$ in this region. However due to the free stream flow causing larger out-of-plane velocities the mean relative uncertainties vary between 2 and $3.3 \%$. These are similar in magnitude to the vertical in-plane uncertainties. The increased uncertainty for the $\mathrm{U}$ and $\mathrm{W}$ velocity components is most likely due to the camera viewing angles used in the experiment. It can be observed in Figure 2 that the cameras have both a horizontal stereo angle applied and a vertical pitch angle to view the laser sheet at the height of the foil tip. It can be seen from Table 2 that the stereo angle between the two cameras is approximately 27 degrees whilst both cameras are pitched down by approximately $25 \mathrm{deg}$. The out of plane uncertainty will depend on the stereo angle used whilst the pitch angle is likely to increase the uncertainty of the vertical velocity component as vertical particle displacements will be observed at a shallower angle then horizontal displacements.

The data acquired at $10 \mathrm{~m} / \mathrm{s}$ shows a significant increase in uncertainty towards the edges of the presented data. This is caused by the low in-plane 
velocities previously discussed, however is exacerbated by too small a $\Delta t$ being set causing very small particle displacements seen in Table 3. This effect was confirmed by comparisons to preliminary data acquired at the intended $\Delta t$ value of $100 \mu \mathrm{s}$ and is presented in Figure 10. However despite improved measurement accuracy near the edges of the field of view increased uncertainty was seen near the vortex structure with the larger $\Delta t$. This highlights the need to optimise the PIV measurement setting depending on the flow features observed. It should also be noted that different seeding levels and camera focus settings were used to obtain this preliminary data. 


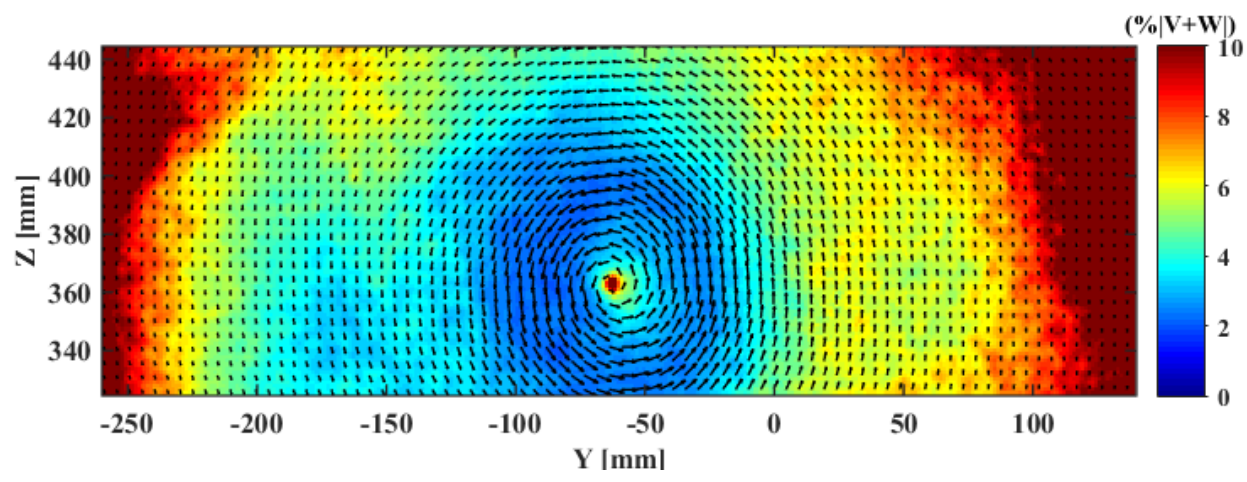

(a) $\Delta t=100 \mu \mathrm{s}$

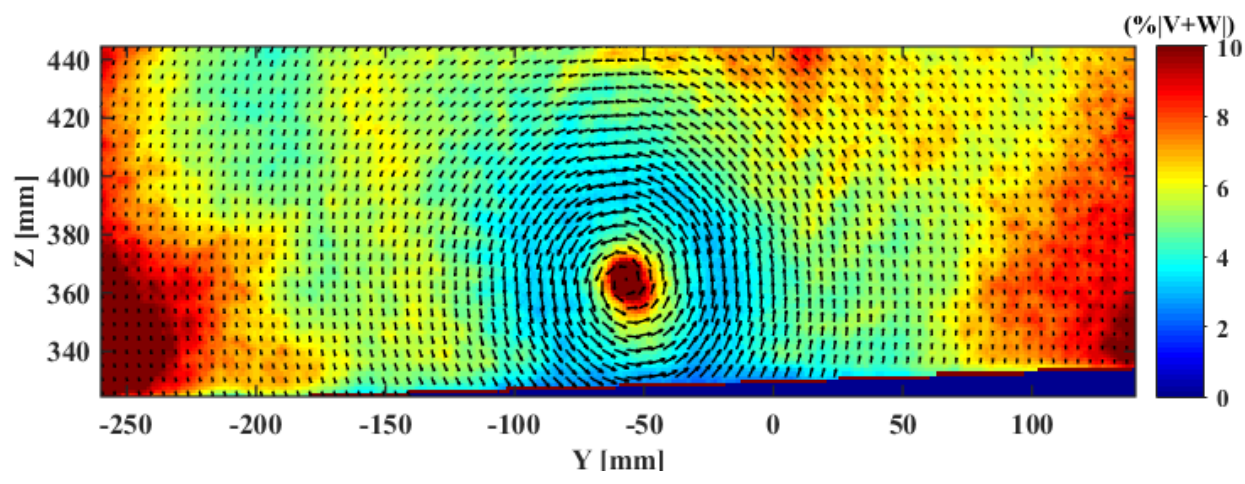

(b) $\Delta t=40 \mu \mathrm{s}$

Figure 10: Vertical in-plane velocity $(\mathrm{W})$ uncertainty with different $\Delta t$ values for $V_{S}=10$ $\mathrm{m} / \mathrm{s}$.

The quantification of the PIV uncertainty provides confidence in the accuracy of the measured velocity field, particularly the in-plane velocities around the vortex structure, where the uncertainty varies between 1.2 and $3.5 \%$ of the in-plane velocity (approximately $0.05-0.1 \mathrm{px}$ ). This appears to be similar to the in-plane velocity uncertainties quoted for other experiments of $0.1 \mathrm{px}$ and 1.5\% (Raffel et al., 2007; De Kat and Van Oudheusden, 2012), whilst larger relative uncertainties are quoted for stereoscopic PIV studies of turbulent shear flows, $3.7 \%$ in-plane and $6.5 \%$ out of plane (Herpin et al., 2008), 
$6 \%$ in-plane and $10 \%$ out of plane (Mullin and Dahm, 2006).The higher uncertainty associated with the centre of the vortex core is likely to be due to a reduction in seeding particles observed in this region associated with particle centrifugation (Raffel et al., 2007). This should not limit the ability of the measurement system to locate the vortex centre, or calculate its structure. This confirms that the measurement accuracy of the PIV system running simultaneously with the DIC system is sufficient to allow coupled FSI experiments to be conducted and the interaction between the structure and the flow field to be quantified.

\section{Benchmark aero-elastic test case}

Having coupled two different acquisition systems together and assessed their uncertainties, it is possible to compare the structural, aerodynamic and fluid results to understand the FSI behaviour of a structure. To provide a case study suitable for validation of FSI simulations, a flexible aerofoil, as described in section 3 , is examined. In order to correctly assess the influence of the coupling between the two non-contact measuring techniques, a steady test case was chosen due to the lower acquisition frequency of the PIV system. An angle of attack $(\alpha)$ of $15^{\circ}$ for a range of wind speeds $\left(V_{S}=0,10\right.$, $15,20,25 \mathrm{~m} / \mathrm{s}$ ) was investigated. In this condition the flow is still attached to the foil and stall does not occur, even at the higher wind speeds. Figure 11 presents the time averaged axial velocity distribution relative to the free-stream velocities at different wind speeds. The time averaged in-plane velocity vectors are displayed and used to locate the position of the vortex centre, highlighted in white. From the figure, it is possible to see that the

vortex core position shifts to the right as the wind speed is increased due to greater structural deformation. 


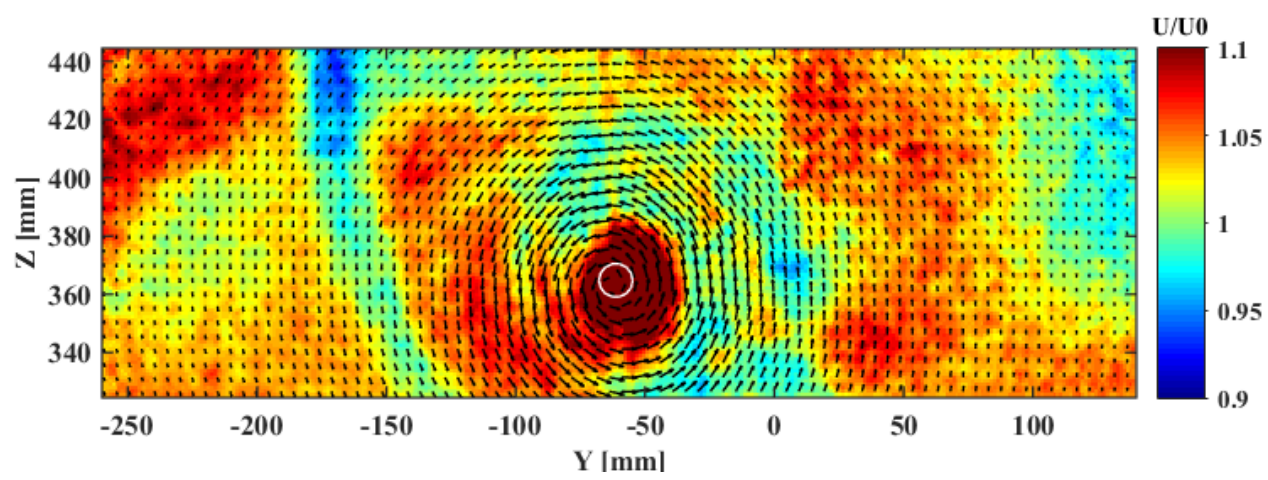

(a) $V_{S}=10 \mathrm{~m} / \mathrm{s}$

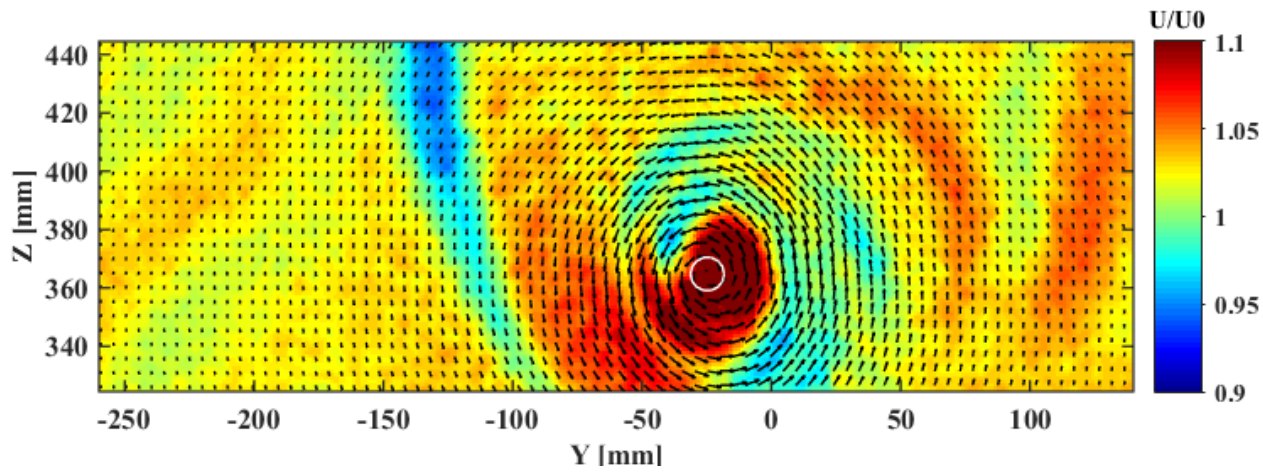

(b) $V_{S}=20 \mathrm{~m} / \mathrm{s}$

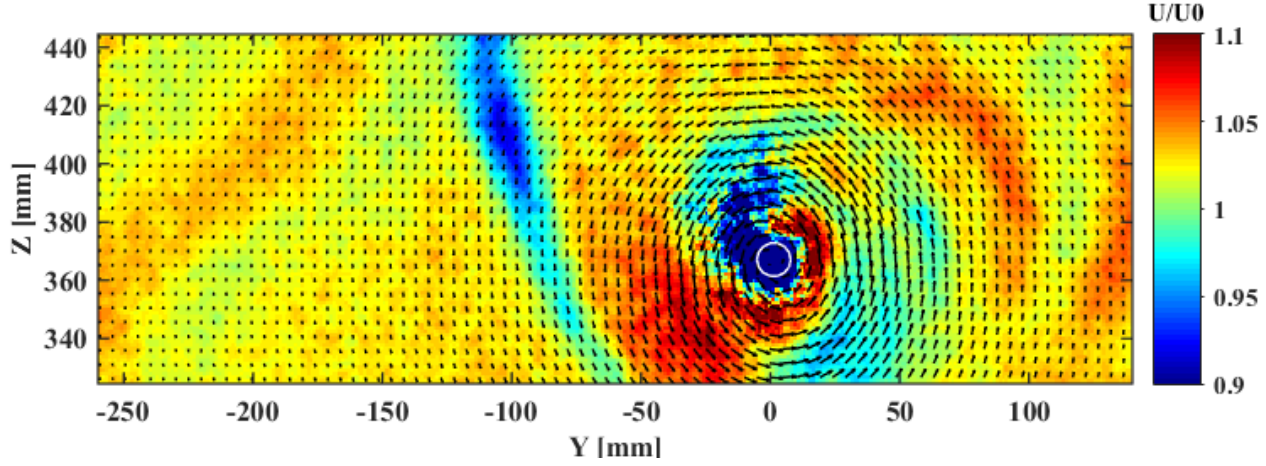

(c) $V_{S}=25 \mathrm{~m} / \mathrm{s}$

Figure 11: Time averaged axial velocity distribution relative to the free stream velocities. The in-plane velocity field is represented as vectors. The vortex centre calculated from the mean velocity field is represented by a white circle. 
The vortex position and the wake wrap-up are presented on a transverse plane one-chord downstream of the aerofoil trailing edge. Figure 11 shows that the vortex core is well formed for $V_{S}=10 \mathrm{~m} / \mathrm{s}$ and $V_{S}=20 \mathrm{~m} / \mathrm{s}$ $(\mathrm{Re}=320000$ and 640000$)$, however, at the higher flow velocity of $V_{S}=25$ $\mathrm{m} / \mathrm{s}(\mathrm{Re}=800000)$ the increased inertial effect moves the wrap-up location further downstream. This increase in Reynolds number with a fixed laser position results in the PIV plane capturing more of the wake features than a fully formed vortex core. It should also be noted that the tested velocity range passes through the critical Reynolds number resulting in the potential for significant flow regime changes as transition from laminar to turbulent flow occurs.

Figure 12(a) presents the structural response of the tip of the aerofoil captured in two different data sets, one where only the DIC system was acquiring (with white light) and one where the two systems were coupled together (with the addition of the magenta filters). The DIC-PIV deflection is averaged over 3000 images, whereas the DIC-only result data is averaged over 130 images. The results show a very good agreement for all the investigated wind speeds, with differences of less than $5 \%$ for wind speeds of $10 \mathrm{~m} / \mathrm{s}$ and less than $2 \%$ for wind speeds greater than $10 \mathrm{~m} / \mathrm{s}$. The standard deviation for all the runs is less than $0.3 \mathrm{~mm}$, recorded at the highest wind speed. The aerodynamic forces normal to the aerofoil are also presented in the Figure 13. Figure $12(\mathrm{~b})$ presents the relative displacement measured from a base wind speed of $10 \mathrm{~m} / \mathrm{s}$ for both the tip deflection and the averaged position of the vortex core. 


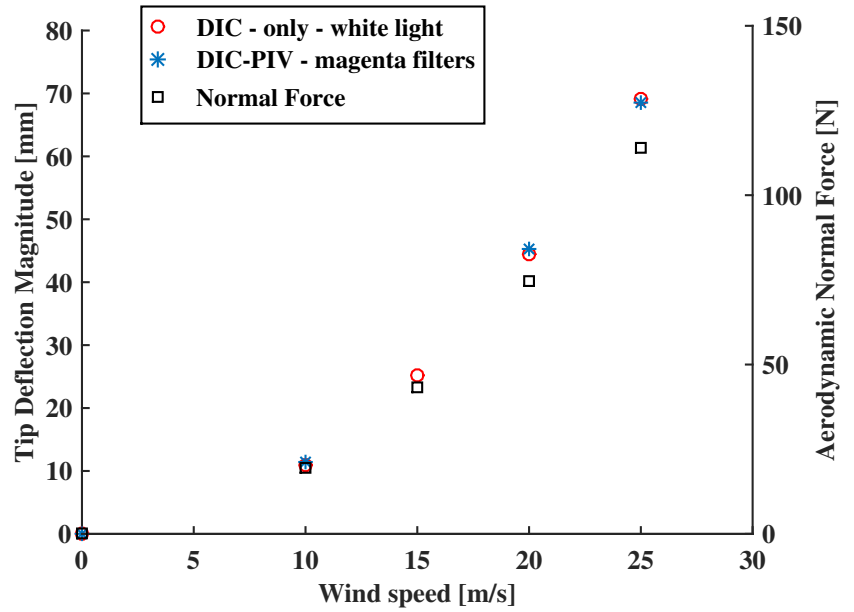

(a) Tip deflection and aerodynamic force.

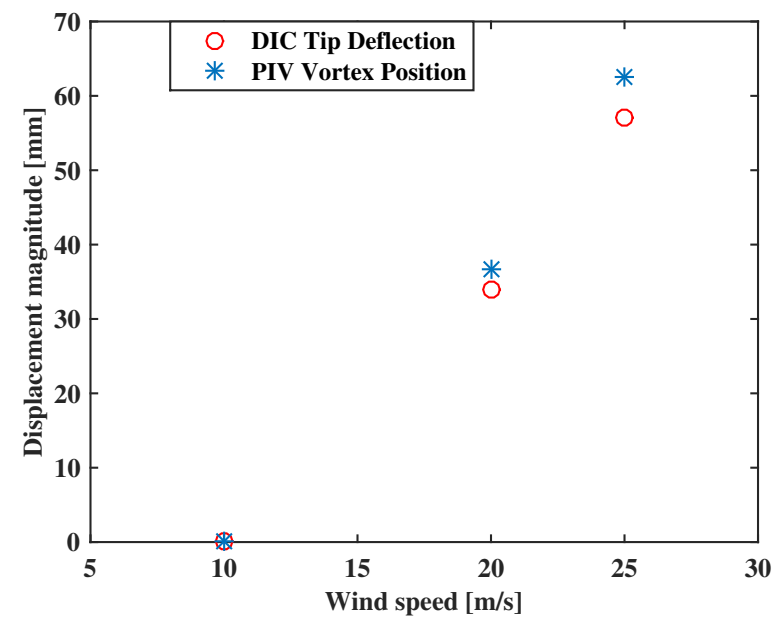

(b) Tip and vortex displacements from $10 \mathrm{~m} / \mathrm{s}$.

Figure 12: Comparison of structural tip displacement between one set of DIC-only data and coupled DIC-PIV data and aerodynamic loads for $\alpha=15$ degrees and a range of wind speeds. 
Considering the highest wind speed of $25 \mathrm{~m} / \mathrm{s}$, the vortex core is displaced by $62.5 \mathrm{~mm}$. The error associated with this measurement will be a combination of the in-plane PIV uncertainty $(2.7 \%)$ and errors associated with the VORTFIND algorithm which should be no more than the PIV vector spacing $(\approx 1.2 \mathrm{~mm})$ (Pashias, 2005). The out-of-plane tip displacement measured by DIC in Figure 12 is $58 \mathrm{~mm} \pm 1.5 \%$, where the accuracy is measured by means of the translation stage (as shown in Figure 4). From these results it is possible to see the close interaction between tip deflection and developed flow features. It is also apparent that the relative displacements of each do not match exactly, demonstrating the complexity involved in aero-elastic problems and the need to understand the interactions more fully. For instance the change in blade twist due to structural deformation and changes in vortex formation due to changing Reynolds number will all affect how the fluid and structure interact and resultant tip vortex roll-up.

Figure 13 presents the change in angle of attack with increasing wind speed due to a difference in the centre of pressure and the shear centre of the structure. The comparison between the averaged results for two sets of DIC measurements (white light) and the coupled PIV-DIC run (magenta filters). The maximum deviation between the two sets of DIC only data was $0.23 \mathrm{~mm}$ in tip motion and $0.07^{\circ}$ in twist change. This demonstrates the repeatability of the technique. The angle of attack decreases with increasing aerodynamic loading reaching a maximum twist deformation of $0.6^{\circ}$. The measured twist angles show similar trends at all span-wise locations. This indicates that the aerodynamic centre of pressure is behind the structural shear centre enabling the aerofoil to delay its stall point as the wind speed increases.

It should be noted that the decrease in the foil angle of attack due to structural deformation has the impact of increasing the lateral displacement of the trailing edge compared to the leading edge. This change in local angle of attack will have the effect of shifting the tip vortex to the right hand side in Figure 11 (i.e. increasing the y position). This helps to explain why the average tip deflection is lower than the tip vortex displacement, as seen in Figure 12(b).

The comparison of the DIC only and coupled data shows a similar trend to the error assessment with the introduction of the magenta filters. The response of the foil structure is very similar, but the results are slightly shifted, most noticeably in twist. The error comparison between the different runs is less than $20 \%$ and the standard deviation of all the measurements is less than $0.02^{\circ}$. 

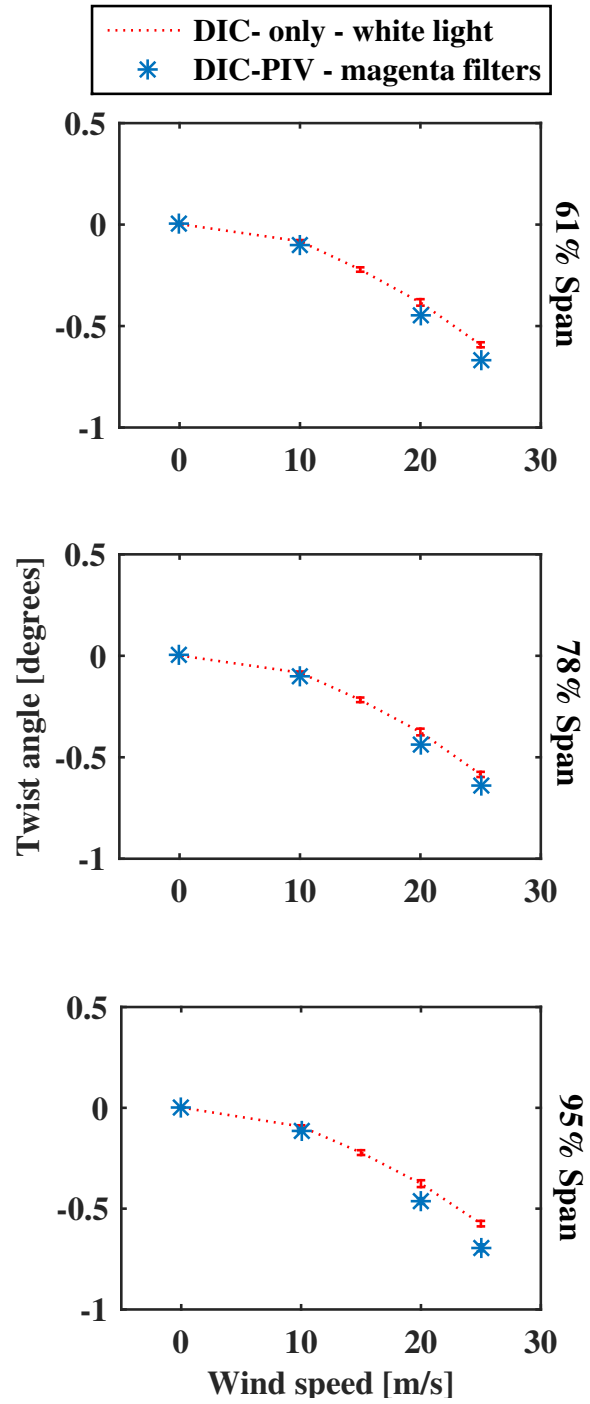

Figure 13: Structural response comparison for the change in angle of attack between DIC only data and coupled DIC-PIV data. The Figure shows the change in twist angle for a range of wind speeds at three different locations in the span-wise direction for $\alpha=15$ degrees.

The results presented in Figure 12 and Figure 13 show the repeatability of the technique and the robustness of the measurement methodology, which is able to capture with very small standard deviations the structural response under fluid load. 


\section{Conclusions}

Two full-field non-contact measurement techniques, PIV and DIC, have been successfully synchronised to provide both structural deformations and fluid response. The impact of coupling the two optical systems on measurement uncertainty has been assessed.

The fluid dynamic and structural response of a flexible composite aerofoil was assessed in the controlled environment of a wind tunnel.

It has been shown that by suitable experimental design the accuracy of the DIC system is not significantly affected by the addition of the PIV to the methodology and vice-versa. The maximum error for DIC measures both in translation and rotation for static and dynamic motions is always less than 5\%. The mean in-plane velocity component uncertainties around the vortex structure varied between $1.2 \%$ and $3.5 \%$ depending on flow speed $(\approx 0.05-0.1 \mathrm{px})$ around the vortex structure. The mean out-of-plane velocity uncertainty around the vortex varied between $2 \%$ and $3.3 \%$ depending on flow speed.

A steady-state test case was described in detail and the structural and fluid deflections were assessed simultaneously. The tip vortex core, which moved by $\approx 62 \mathrm{~mm}$ at the highest wind speed, is directly compared to the deformation of the structure, which deflected by $\approx 58 \mathrm{~mm}$. This corresponded to a maximum foil twist of $\approx 0.6 \mathrm{deg}$. The example results show the interaction between the structural response and the corresponding fluid field. The methodology was shown to be robust and provided repeatable results with low standard deviation.

By demonstrating and quantifying the relationship between structural and fluid response, the presented methodology has the potential to investigate the influence of changing the internal design of a structure to evoke deformations that have a positive effect on fluid response, i.e. passive adaptation. Therefore, this technique will be relevant to a large range of applications not only as an experimental method, but also to provide valuable validation cases for numerical FSI investigations. 


\section{Acknowledgement}

The authors would like to acknowledge the EPSRC for funding this re-

search under the grant number EP/I009876/1 as well as providing the Photron SA3 high speed cameras under loan from the Engineering Instrument Pool (EIP). The authors would also like to thank David Marshal and his team in the R.J. Mitchell wind tunnel, the members of the Testing and Structures Research Laboratory at the University of Southampton. In addition, our thanks to David Hollis from LaVision for assistance with the DIC and PIV methodologies and for providing the High Speed controller that enabled the coupling between the two measuring systems.

\section{References}

Banks, J., Marimon Giovannetti, L., Soubeyran, X., Wright, A. M., Turnock, S. R., Boyd, S. W., 2015. Assessment of Digital Image Correlation as a method of obtaining deformations of a structure under fluid load. Journal of Fluids and Structures 58, 173-187.

Castro, I. P., 2001. Calibration tests in the working section of the R J Mitchell Wind Tunnel. Tech. rep., University of Southampton.

Chen, F., Chen, X., Xie, X., Feng, X., Yang, L., Sep. 2013. Full-field 3D measurement using multi-camera digital image correlation system. Optics and Lasers in Engineering 51 (9), 1044-1052.

Crammond, G., Boyd, S., Dulieu-Barton, J., Dec. 2013. Speckle pattern quality assessment for digital image correlation. Optics and Lasers in Engineering 51 (12), 1368-1378.

de Borst, R., Nithiarasu, P., Tezduyar, T., Yagawa, G., Zohdi, T., 2013. Computational Fluid-Structure Interaction. John Wiley \& Sons, Ltd., Publication.

De Kat, R., Van Oudheusden, B. W., 2012. Instantaneous planar pressure determination from PIV in turbulent flow. Experiments in Fluids 52 (5), 1089-1106.

Ducoin, A., André Astolfi, J., Sigrist, J. F., 2012. An experimental analysis of fluid structure interaction on a flexible hydrofoil in various flow regimes 
including cavitating flow. European Journal of Mechanics, B/Fluids 36, 63-74.

Fedorov, V., 2012. Bend-Twist Coupling Effects in Wind Turbine Blades. Ph.D. thesis, Technical University of Denmark.

Funatani, S., Fujisawa, N., Ikeda, H., 2004. Simultaneous measurement of temperature and velocity uising two-colour LIFT combined with PIV with a colour CCD camera and its application to the turbulent buoyant plume. Measurement Science and Technology 15, 983-990.

Grant, B. M. B., Stone, H. J., Withers, P. J., Preuss, M., 2009. Hightemperature strain field measurement using digital image correlation. Strain Analysis 44 (6), 263-271.

Guo, X., Liang, J., Tang, Z., Cao, B., Yu, M., 2014. High-temperature digital image correlation method for full-field deformation measurement captured with filters at $2600^{\circ} \mathrm{C}$ using spraying to form speckle patterns. Optical Engineering 53 (6).

Gurit, 2015a. SA 80 Toughened epoxi adhesive film. Tech. rep.

Gurit, 2015b. SE 84 LV Low temperature cure epoxy prepreg. Tech. rep.

Herpin, S., Wong, C. Y., Stanislas, M., Soria, J., 2008. Stereoscopic PIV measurements of a turbulent boundary layer with a large spatial dynamic range. Experiments in Fluids 45 (4), 745-763.

Hou, G., Wang, J., Layton, A., 2012. Numerical Methods for Fluid-Structure Interaction - A Review. Communications in Computational Physics 12 (2), $337-377$.

Jones, G. S., Lin, J. C., Allan, B. G., Milholen, W. E., Rumsey, C. L., Swanson, R. C., 2008. Overview of CFD Validation Experiments for Circulation Control Applications at NASA. In: International Powered Lift Conference. pp. 1-16.

LaVision, 2015. PTU X High-Speed. Tech. rep.

LaVision, 2016. Flow master. Tech. rep. 
Malijaarsl, P. J., Kaminski, M. L., 2015. Hydro-elastic Analysis of Flexible Propellers: an overview. In: Fourth International Symposium on Marine Propulsors. Austin.

Mccormick, N., Lord, J., 2012. Digital image correlation for structural measurements. In: Proceedings of the Institution of Civil Engineers. Vol. 165. ICE Publishing, pp. 185-190.

Mullin, J. A., Dahm, W. J. A., 2006. Dual-plane stereo particle image velocimetry measurements of velocity gradient tensor fields in turbulent shear flow. I. Accuracy assessments. Physics of Fluids 18 (3).

Note, P.-j., 2014. Development of a twist sensitive daggerboard for wind tunnel testing. Msc thesis, University of Southampton.

Pan, B., Wu, D., Yu, L., 2012. Optimization of a three-dimensional digital image correlation system for deformation measurements in extreme environments. Applied optics 51 (19), 4409-19.

Pashias, C., 2005. Propeller tip vortex simulations using adaptive grid refinement based on flow feature identification. Ph.d thesis, University of Southampton.

Pemberton, R., Turnock, S. R., Dodd, T., Rogers, E., 2002. A novel method for identifying vortical structures. Journal of Fluids and Structures 16 (23), $1051-1057$.

Phillips, A. B., Turnock, S. R., Apr. 2013. Application of the VORTFIND algorithm for the identification of vortical flow features around complex three-dimensional geometries. International Journal for Numerical Methods in Fluids 71 (11), 1461-1474.

Post, M. E., Trump, D. D., Goss, L. P., Hancock, R. D., 1994. Two-color particle-image velocimetry using a single argon-ion laser. Experiments in Fluids 16, 263-272.

Prasad, A., Jensen, K., 1995. Scheimpflug stereocamera for particle image velocimetry in liquid flows.

Prasad, A. K., 2000. Stereoscopic particle image velocimetry. Experiments in Fluids 29 (February), 103-116. 
Raffel, M., Willert, C., Wereley, S., Kompenhans, J., 2007. Particle Image Velocimetry - A Practical Guide.

Rastogi, P. K., Erwin, H., 2012. Optical Methods for Solid Mechanics: A Full-Field Approach.

Reu, P., 2012. Stereo-Rig Design : Creating the Stereo-Rig Layout - Part 1. Experimental Techniques 36 (Dic), 3-4.

Schreier, H., Orteu, J.-J., Sutton, M. a., 2009. Image Correlation for Shape, Motion and Deformation Measurements: Basic Concepts, Theory and Applications. No. Dic. Springer US, Boston, MA.

Sciacchitano, A., Neal, D. R., Smith, B. L., Warner, S. O., Vlachos, P. P., Wieneke, B., Scarano, F., 2015. Collaborative framework for piv uncertainty quantification: comparative assessment of methods. Measurement Science and Technology 26 (7), 074004.

Siddiqui, M. Z., 2014. 2D-DIC for the quantitative validation of FE simulations and non-destructive inspection of aft end debonds in solid propellant grains. Aerospace Science and Technology 39, 128-136.

Tang, Z., Liang, J., Xiao, Z., Guo, C., Feb. 2012. Large deformation measurement scheme for 3D digital image correlation method. Optics and Lasers in Engineering 50 (2), 122-130.

Wang, Y. Q., Sutton, M. A., Ke, X. D., Schreier, H. W., Reu, P. L., Miller, T. J., Feb. 2011. On Error Assessment in Stereo-based Deformation Measurements. Experimental Mechanics 51 (4), 405-422.

Wieneke, B., 2015. Piv uncertainty quantification from correlation statistics. Measurement Science and Technology 26 (7), 074002. 